\title{
Research of airport avian information forecasting System Based on Multi-Agent
}

\author{
Liang Zhang, a , Na Lu' ${ }^{2, b, ~ * ~}$ \\ ${ }^{1}$ Airport Operation Management Department,Guangzhou Civil Aviation College Guangzhou \\ 510403, China; \\ ${ }^{2}$ College of Economics and Management, Xi'an Aeronautical University, Xi'an710077, China; \\ a25267764@qq.com, bhr.luna@163.com, \\ *corresponding author
}

Keywords: Airport, Bird-strike, Multi-Agent, Cooperation mechanisms, Prediction and Forecast.

\begin{abstract}
Airport avian information forecasting as a synthetic evaluation system, is provided with general characteristics of complexity. In traditional requirement analysing technology (objectoriented and structure-oriented), it is hard to get a model to describe system behaviour and information exchange. By employing the design thought of multi-agent and the object-driven process modelling method, the paper first gives the configuration of the airport avian information forecasting System Based on Multi-Agent and describes the function of each single agent. Then the authors put forward the ways of working and the cooperation mechanisms of agents. This research fills the gaps in the domestic research, which provides the theoretical basis for the design to the airport avian information forecasting System.
\end{abstract}

\section{Introduction}

Bird strike (also known as bird impact), which is accompanied by the born of the aviation industry, has caused airlines substantial damage on engines and airframes. With the rapid development of aviation industrial and the increasing awareness of the ecological environment protection by the government, bird strike accident emerges one after another incessantly, which severely influence the flight safety [1-2].Based on the survey and research data of avian information, food resources and the ecological environment of habitat, combined with the weather condition, we can predict the probability of bird strike, which is the core of the airport avian information Prediction and Forecast. Due to the complexity and disreputability of Airport avian information forecast, it is hard to get a model to describe system behavior and information exchange in traditional requirement analyzing technology (object-oriented and structure-oriented). By employing the design thought of multi-agent and the object-driven process modeling method, airport avian information forecasting System has been discussed and designed aiming at reduce the probability of bird strike, which can also provide the theories foundation for the future intelligent airport management. So in order to optimize the process of the bird strike prevention strategy and macroeconomic management, first the paper gives the configuration of the airport avian information forecasting System Based on Multi-Agent and then describes the function of each single agent then put forward the ways of working and the cooperation mechanisms of agents.

\section{The Theory of Multi-Agent System}

A multi-agent system (M.A.S.) is a computerized system composed of multiple interacting intelligent agents within an environment. Multi-agent systems can be used to solve problems that are difficult or impossible for an individual agent or a monolithic system to solve. Intelligence may include some methodic, functional, procedural approach, algorithmic search or reinforcement 
learning. The terminology of ABM tends to be used more often in the sciences, and MAS in engineering and technology. [3] Topics where multi-agent systems research may deliver an appropriate approach include online trading, [4] disaster response, [5,6] and modeling social structures. [7] Agents can be divided into different types ranging from simple to complex. Some categories suggested to define these types include: Passive agents [8] or agent without goals (like obstacle, apple or key in any simple simulation). Active agents with simple goals (like birds in flocking, or wolf-sheep in prey-predator model) Cognitive agents (which contain complex calculations)

\section{Configuration of the Airport Avian Information Forecasting System}

In this paper, the layer structure was adopted to design the airport avian information forecasting System. The manageable units of the airport avian information forecasting System is regarded as a single agent with intelligence. Data communication can be carried out among the agents. Upper agent monitors the operation of the lower level. Usually each agent run stand-alone, the upper agent interferes in the lower level only in exceptional circumstance.

i. The central agent structure of the national airport avian information forecasting System

The central agent structure of the national airport avian information forecasting system is responsible for monitoring the avian information, bird migration route and migration laws of birds of the whole nation, predicting when to arrive over the aerodrome and the scale of the birds. Meanwhile, according to the awareness of itself, the central agent structure of the national airport avian information forecasting System direct and coordinate the lower level agent about the avian information forecast task, which allow the Administrative staff to correct and interpose the process of forecast. The central agent structure of the national airport avian information forecasting System listed in Fig.1.

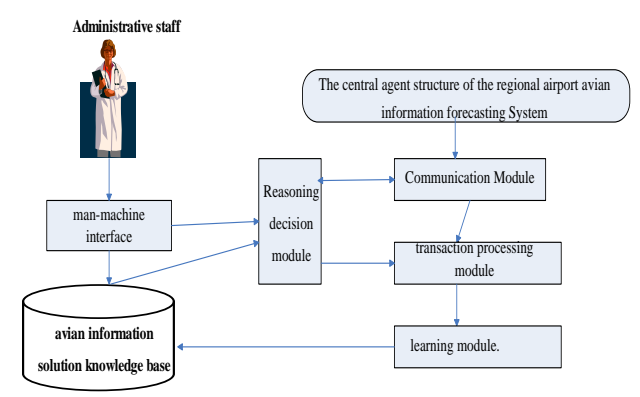

Fig 1. The central agent structure of the national airport avian information forecasting System

ii. The central agent structure of the regional airport avian information forecasting System

The central agent structure of the regional airport avian information forecasting System is responsible for monitoring the avian information, bird migration route, migration laws of birds of their own reign and direct and coordinate the lower level agent about the avian information forecast task under the guidance of the upper level. Meanwhile, the central agent structures of the regional airport avian information forecasting System receive instructions released by the upper level and coordinate among the different region agent, which ensure that all the data sources have been equally accessed. The central agent structure of the regional airport avian information forecasting System listed in Fig 2 


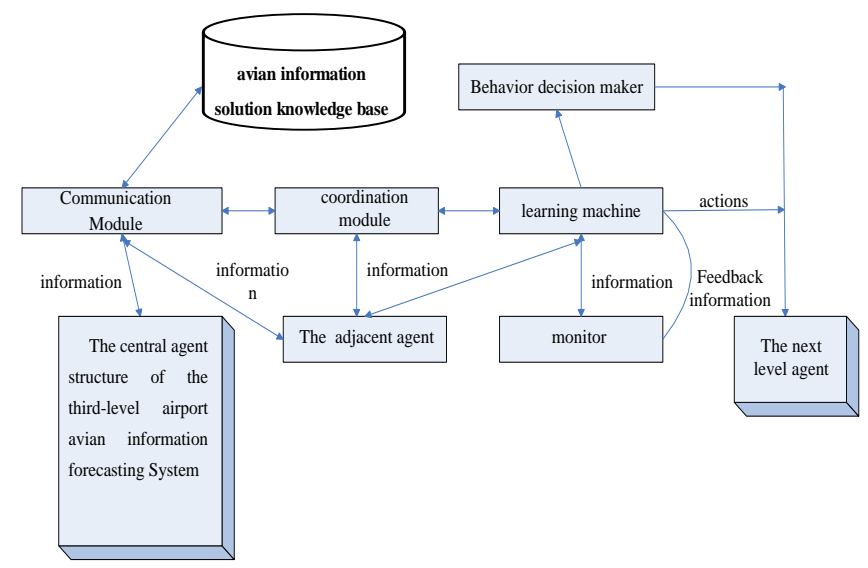

Fig 2. The central agent structure of the regional airport avian information forecasting System

iii. The central agent structure of the third-level airport avian information forecasting System

The central agent structure of the third-level airport avian information forecasting System is responsible for cooperating with bird strike prevention staff of their own region to reduce the probability of bird strike. The whole region is divided into small regions, in which there is an avian information forecasting agent responsible for dealing with the avian information forecast and prediction. The central agent structure of the third-level airport avian information forecasting System listed in Fig.3.

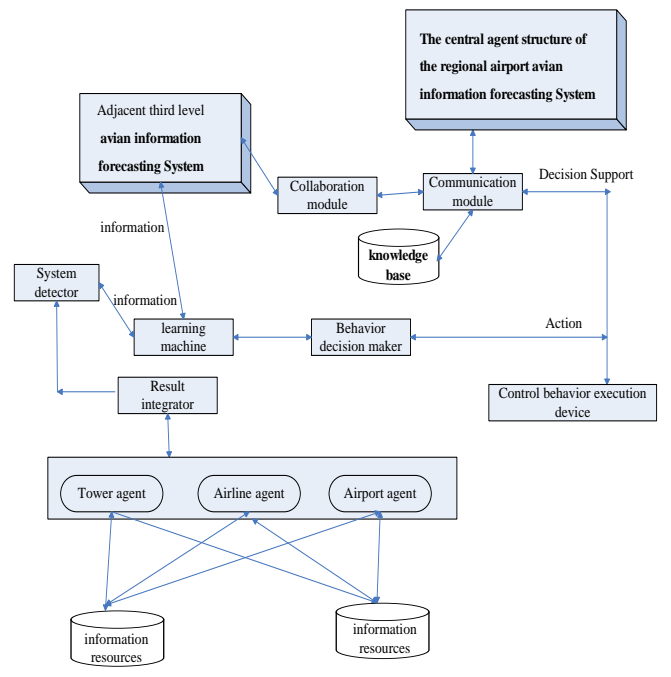

Fig 3. The central agent structure of the third-level airport avian information forecasting System

\section{Teamwork mode in Multi-Agent Airport Avian Information Forecasting System}

The central agent structure of the national airport avian information forecasting system is responsible for monitoring the avian information, bird migration route and migration laws of birds of the whole nation. The central agent structure of the regional airport avian information forecasting System sends message to the national agent every now and again. The central agent structure of the national airport avian information forecasting system makes full use this information and itself own knowledge to analysis regional airport avian information, predicting when to arrive over the destination aerodrome and the scale of the birds, which provides control directive to the regional agent when necessary. The regional agent must execute these control directives unconditionally and feedback the control effect to the central agent structure of the national airport avian information forecasting system, which allow it to update the empirical knowledge. When necessary, the central agent structure of the national airport avian information forecasting system will control the whole forecasting system by Man-Machine Interface. 
The process of commanding and controlling for the central agent structure of the regional airport avian information forecasting System can be described as follow: The detector of the agent sends the avian information of its region to the learning models of the agent, meanwhile, the adjacent agent of the forecasting system also provide avian information to the learning models of the agent; the learning models of the agent provides decisions to the decision-making system of the agent according to the received information and its own experience knowledge; the decision-making system chooses behavior which is performance by the control system. When the control behavior is inconsistent with the actual information, the agent will modify the predicting information. After each certain period of time, the detector will send the avian information to the region agent again and send a reinforcement signal to the learning models of the agent. The learning model of the agent revises the decision information, and then act upon the avian information forecasting system. The above process is an iterative process.

When the regional agent finds it is necessary to inform the avian information to the adjacent airport, the regional agent can coordinate with adjacent agent by the help of coordination module. The central agent structure of the national airport avian information forecasting System can send control directives to the regional agent by communication module and the agent must execute the control directives unconditionally; the agent must feedback the control effect to the national agent to refresh itself experience knowledge. When executing the directives send by the national agent, the learning models of the reginal agent suspend operation.

In the central agent structure of the third-level airport avian information forecasting System, the large region is divided into a small series of regions, there is a forecasting agent in each agent, meanwhile, tower, airport and airline in this region are regarded as a single agent, theses agent integrate data by calculating-result models to obtain the synthetic evaluation of the region. Meanwhile, this information will be send to the learning models, which provides decision-making basis for the decision-making machine. The decision-making machines select behavior and execute control behavior.

\section{Conclusion}

Based on the theory of multi-agent system, the paper proposed the configuration of airport avian information forecasting System and each sub-system, meanwhile the paper also put forward the teamwork mode of the system, which provides new ideas to the airport avian forecast.

\section{References}

[1]. X. L. Wang, Q. R. Yang, J. M. Liu, et al., Study on the ecology of the bird in the Tianhe Airport, J. Cent. China Normal Univ. (Nat. Sci.)33(4) (1999)579-583

[2]. E. C. Cleary, R.A Dolbeer, Wildlife Hazard Management at Airports: A Management for Airport Personnel, Federal Aviation Administration, Office of Airport Safety and Standards, Washington,2005.

[3]. Niazi, Muaz; Hussain, Amir (2011). "Agent-based Computing from Multi-Agent Systems to Agent-Based Models: A Visual Survey" (PDF). Scientometrics. Springer. 89 (2): 479-499. doi:10.1007/s11192-011-0468-9.

[4]. Rogers, Alex; David, E.; Schiff, J.; Jennings, N.R. (2007). "The Effects of Proxy Bidding and Minimum Bid Increments within eBay Auctions". ACM Transactions on the Web.

[5]. Schurr, Nathan; Marecki, Janusz; Tambe, Milind; Scerri, Paul; Kasinadhuni, Nikhil; Lewis, J.P. (2005). "The Future of Disaster Response: Humans Working with Multiagent Teams using DEFACTO" (PDF).

[6]. Genc, Zulkuf; et al. (2013). "Agent-based information infrastructure for disaster management]" (PDF). Intelligent Systems for Crisis Management: 349-355. 
[7]. Sun, Ron; Naveh, Isaac. "Simulating Organizational Decision-Making Using a Cognitively Realistic Agent Model". Journal of Artificial Societies and Social Simulation.

[8]. Kubera, Yoann; Mathieu, Philippe; Picault, Sébastien (2010), "Everything can be Agent!" (PDF), Proceedings of the ninth International Joint Conference on Autonomous Agents and MultiAgent Systems (AAMAS'2010), Toronto, Canada: 1547-1548. 